\title{
A Magnetic Model for Low/Hard States Associated with Jets in Black Hole X-Ray Binaries
}

\author{
Jiuzhou Wang ${ }^{1}$, Dingxiong Wang ${ }^{1}$ Zhaoming Gan ${ }^{2}$, Changyin Huang ${ }^{1 *}$ \\ ${ }^{1}$ School of Physics, Huazhong University of Science and Technology, Wuhan, China \\ ${ }^{2}$ Shanghai Astronomical Observatory, Chinese Academy of Sciences, Shanghai, China \\ Email: *dxwang@mail.hust.edu.cn
}

Received April 19, 2012; revised May 22, 2012; accepted May 30, 2012

\begin{abstract}
A model for the low/hard (LH) state associated with a steady jet in black hole X-ray binaries (BHXBs) is proposed based on disc-corona model with open magnetic fields trapped in magnetic patches, which arises from "flux expulsion" effect of convective turbulence. We fit the spectral profiles of the LH state for the BHXBs, 4U 1543-475, GX 339-4, XTE J1550-564 and GRO J1655-40, and fit the relation between jet power and X-ray luminosity dynamically in the LH state by adjusting accretion rate and the outer boundary of the corona over the disc.
\end{abstract}

Keywords: Accretion; Accretion Disks; Black Hole Physics; X-Rays; Binaries; Magnetic Fields

\section{Introduction}

Spectral states observed in black hole X-ray binaries (BHXBs) involve a number of unresolved issues in astrophysics, displaying complex variations not only in the luminosities and energy spectra, but also in presence/ absence of jets and quasi-periodic oscillations (QPOs).

Belloni [1] classified the states of BHXBs as low/hard (LH) state, hard intermediate state (HIM), soft intermediate (SIM) state and high/soft (HS) state, which display different luminosity and hardness associated with different behavior of QPOs and radio loudness. McClintock \& Remillard [2] used four parameters to define X-ray states based on the very extensive RXTE data archive for BHXBs, in which three states, i.e., thermal-dominant (TD) state, low/hard (LH) state and steep power law (SPL) state are included. In TD state, the flux is dominated by the thermal radiation from the inner accretion disk, and QPOs are absent or very weak. The spectral profile of LH state is characterized by a hard power law component at $2-20 \mathrm{keV}$ with a photon index $\Gamma$ in the range $\approx 1.4-2$ and an exponential cut-off at about 100 $\mathrm{keV}$, which is associated with a quasi-steady radio jet. SPL state has a strong power-law component with $\Gamma \sim 2.5$, which is associated with high-frequency QPOs. Although a consensus on classification of spectral states of BHXBs has not been reached, it is widely accepted that these states can be reduced to only two basic states, i.e., a hard state and a soft one, and jets can be observed in hard states, but cannot be in soft states.

"Corresponding author.
Just like classification of spectral states agreement has not been reached on modeling the spectral states of BHXBs. The accretion flow in LH state is usually supposed to be a truncated thin disk with an inner advection-dominated accretion flow (ADAF) in the prevailing scenario [2-5], and henceforth this model is referred to as truncated ADAF model. Generally speaking, the thermal component of the spectra of BHXBs can be well fitted by a standard thin accretion disk, while the non-thermal component can be interpreted by invoking thermal Comptonization, which originates from either a hot disk (self-Compton process in ADAF) or disk-corona (external Compton process). Although similar spectral profiles of BHXBs can be fitted either by disk-corona model or by truncated ADAF model, there exists an essential difference between the two types of models, i.e., the cold disk remains at or near to the innermost stable circular orbit (ISCO) in disk-corona model, not appearing to truncate beyond ISCO as in ADAF model.

Disk-corona model seems to be supported by a number of recent observations. For example, XMM-Newton observations of GX 339-4 show that a broad iron line together with a dim, hot thermal component was present in its spectra during the hard state. This effect seems to be observed in a few other sources such as Cygnus X-1, and SWIFT J1753.5-0127 [6,7]. Recently, Reis, Miller \& Fabian [8] studied the Chandra observation of XTE $\mathrm{J} 1118+480$ in the canonical LH state, and a thermal disk emission with a temperature of approximately $0.21 \mathrm{keV}$ is found at greater than the 14 level, and they concluded that this thermal emission most likely originates from an 
accretion disk extending close to ISCO. The results of fits made to both components strongly suggest that a standard thin disk remains at or near to ISCO, at least in bright phases of the LH state. Very recently, Reis, Fabian \& Miller [9] presented an X-ray study of eight black holes in the LH state, and they found that a thermal disk continuum with a color temperature consistent with $L \propto T^{4}$ is clearly detected in all eight sources and the detailed fits to the line profiles exclude a truncated disk in each case.

One of the main characteristics of the LH state of BHXBs is its association with quasi-steady jets. Although different mechanisms have been proposed for interpreting jet production in black hole systems of different scales, such as the plasma gun [10], the cosmic battery [11] and the magnetic tower [12], the most promising mechanism for powering jets is magneto-centrifugal mechanism, which relies on an accretion disk threaded by a poloidal, large-scale magnetic field [13-16].

Recently, Gan et al. [17] proposed a disk-corona model for BHXBs, in which the closed magnetic field lines pipe the hot matter evaporated from the disk, and shape it in the corona above the disk to form a magnetically induced disk-corona system. Later, we combined epicyclic resonances with the disk-corona model given by [17] to interpret the high frequency QPOs with 3:2 pairs observed in SPL states of the three BHXBs, i.e., GRO J1655-40 (450, 300Hz), XTE J1550-564 (276, 184Hz) and GRS 1915+105 (168, $113 \mathrm{~Hz})$ [18]. Although some spectral profiles have been fitted in the LH state, a clear association with a quasi-steady jet has not been revealed, and this becomes a great challenge to the present theoretical models.

We notice that corona is a perfect launching site for outflow from accretion disk [19], and disk-corona model provides a possible scenario for interpreting how matter loading into a jet occurs. In this paper, we intend to model the LH state of BHXBs based on a disk-corona model, in which the inner edge of the accretion disk is assumed to extend to ISCO, and the jets are driven by the patched open magnetic field suggested by Spruit \& Uzdensky [20]. The reason of taking this kind of magnetic field structure lies in the following aspects. 1) The magnetic patches reduce reconnection across the disk and thus reduce the outward diffusion of magnetic field; 2) The magnetic patches lose angular momentum effectively and thus make the magnetic field drift inward; 3 ) The trapped magnetic flux plausibly varies on timescales of days or longer, corresponding to the time for accretion from the outer edge of the disk, and thus is consistent with the timescales of the state transitions in BHXBs; and 4) The jet can be driven effectively by the patched open magnetic field, and the disk luminosity can be lowered remarkably due to the transfer of energy of accreting matter into the jet.

This paper is organized as follows. In Section 2, we present a detailed description of our model, in which the jet is driven by the patched open magnetic field, and the coupling of the jet with accretion process is taken into account based on the conservation of energy and angular momentum. In Section 3, the spectral profiles of the LH state of five BHXBs are fitted based on the disk-corona model, and the relation between jet power and X-ray luminosity is fitted by adjusting accretion rate and the outer boundary of the corona over the disk. In Section 4 we propose a scenario of state transitions in BHXBs, in which a correlation of magnetic field configuration with the transition from hard to soft states is discussed. In addition, we suggest that the evolution of the magnetic field configuration in black hole accretion disk can be regarded as the second parameter in the state transitions of BHXBs. Finally, in Section 5, we discuss some issues related to our model.

Throughout this paper the geometric units $\mathrm{G}=\mathrm{c}=1$ are used.

\section{Description of Our Model}

In order to interpret the association of the $\mathrm{LH}$ state of BHXBs with a quasi-steady jet, we take the scenario of magnetic patches suggested by [20], i.e., a large portion of the net vertical magnetic flux threading a disk gets concentrated into patches of strong field due to "flux expulsion" effect of convective turbulence [21,22]. A schematic drawing of disk-corona model with magnetic patches is shown in Figure 1.

The configuration given in Figure $\mathbf{1}$ has some advantages on interpreting the LH state of BHXBs. 1) The outward diffusion of trapped fields by turbulent reconnection can be reduced significantly once the magnetic field locally becomes strong enough to avoid being tangled, and the jet can be driven effectively by the open large-scale magnetic field concentrated in the patches; 2) The disk luminosity can be depressed effectively due to part of energy is transferred into the jet; 3) The corona geometry is scarcely affected by the magnetic patches, which distribute dispersedly on the disk and occupy a small fraction of the disk surface; and 4) The large scale patched open magnetic field is apt to collimate the jet from black hole accretion disk. Thus the LH state of BHXBs associated with a quasi-steady jet can be fitted based on disk-corona model with the magnetic patches.

As argued in [20], the magnetic field strength $B_{\mathrm{p}}$ inside the patches is estimated by

$$
B_{\mathrm{p}} \approx \sqrt{8 \pi P_{\mathrm{gas}} / \beta_{\mathrm{MRI}}}
$$

where $\beta_{\mathrm{MRI}}=\mathrm{O}(1)$ is a parameter related to magnetorotational instability (MRI), and $P_{\text {gas }}$ is the gas pressure 


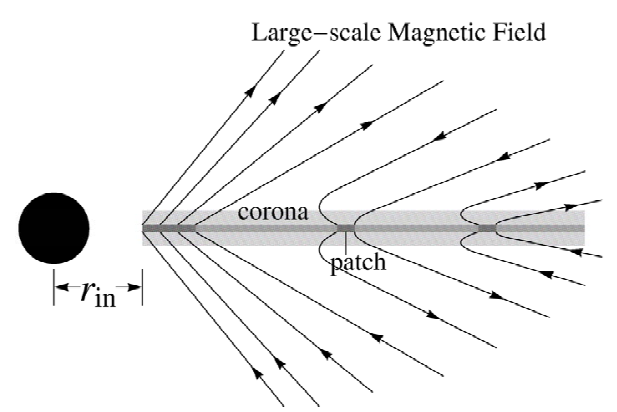

Figure 1. A schematic drawing of disc-corona model with magnetic patches.

dominating over the radiation pressure inside the disk. According to Balbus \& Hawley [23], the interior viscous process is dominated by tangled small-scale magnetic field, $B_{\mathrm{D}}$, and the viscous pressure is comparable to magnetic pressure, and thus we assume

$$
-t_{r \varphi}=\alpha P_{\mathrm{gas}} \sim P_{\mathrm{mag}}=B_{\mathrm{D}}^{2} / 8 \pi .
$$

In this paper, we introduce two parameters, $\lambda_{r}$ and $\lambda_{\varphi}$, to describe the distribution of magnetic patches in the disk as follows,

$$
\left\{\begin{array}{l}
\mathrm{d} r_{\mathrm{p}}=\lambda_{r} \mathrm{~d} r \\
\mathrm{~d} \varphi_{\mathrm{p}}=\lambda_{\varphi} \mathrm{d} \varphi
\end{array}\right.
$$

In Equation (3) the radial coordinate of the patch $r_{\mathrm{p}}$ is related to the disk radius $r$ by the radial fraction factor $\lambda_{r}$, and the toroidal coordinate of the patch $\varphi_{\mathrm{p}}$ to the toroidal coordinate $\varphi$ of the disk by the toroidal fraction factor $\lambda_{\varphi}$. Henceforth the subscript "p" represents the quantities related to the magnetic patches. Thus the magnetic torque exerted on the magnetic patches is given by

$$
\mathrm{d} \tau_{\mathrm{p}}=\frac{\gamma}{2 \pi} r B_{\mathrm{p}}^{2} \mathrm{~d} A_{\mathrm{p}},
$$

where $\mathrm{d} A_{\mathrm{p}}=r \mathrm{~d} r_{\mathrm{p}} \mathrm{d} \varphi_{\mathrm{p}}$ is the area element of the magnetic patch, and $\gamma$ is the ratio of the toroidal component $\left|B_{\varphi, \mathrm{p}}\right|$ to $B_{\mathrm{p}}$. The jet power driven by each magnetic patch is

$$
\mathrm{dP}_{\mathrm{p}}=\Omega_{\mathrm{p}} \mathrm{d} \tau_{\mathrm{p}},
$$

where $\Omega_{\mathrm{p}}$ is the average angular velocity of magnetic patches. Since the magnetized patch drifts inward with a speed greater than that of accreting matter, $\Omega_{\mathrm{p}}=k \Omega_{\mathrm{K}}$ should be less than the Keplerian angular velocity, and $k$ is a factor less than unity.

Incorporating Equations (2)-(5), we have the jet power within the radius $r$ driven by the open magnetic field of all patches as follows,

$$
\mathrm{P}_{\mathrm{p}}(r)=\int \mathrm{dP}_{\mathrm{p}} \approx f_{\mathrm{p}} \int_{r_{\text {in }}}^{r} \Omega_{\mathrm{K}} B_{\mathrm{p}}^{2} r^{2} \mathrm{~d} r,
$$

where four parameters $k, \gamma \lambda_{r}$, and $\lambda_{\varphi}$ are incur- porated into $f_{\mathrm{p}}=k \gamma \lambda_{r} \lambda_{\varphi}$ with $\beta \approx 1$.

The flux of angular momentum $H_{\mathrm{p}}$ transferred from the disk into the jet is related to the flux of energy $S_{\mathrm{p}}$ by $H_{\mathrm{p}}=S_{\mathrm{p}} / \Omega_{\mathrm{K}}$, and $S_{\mathrm{p}}$ can be calculated by

$$
S_{\mathrm{p}} \equiv(4 \pi r)^{-1}\left(\mathrm{dP}_{\mathrm{p}} / \mathrm{d} r\right),
$$

Incorporating Equations (1), (6) and (7), we have

$$
H_{\mathrm{p}}=2 f_{\mathrm{p}} r P_{\text {gas }} .
$$

Since $d \mathrm{P}_{\mathrm{p}}$ is the jet power driven by the magnetic patches in the ring of width $\mathrm{d} r$, the quantities $S_{\mathrm{p}}$ and $H_{\mathrm{p}}$ are respectively the average fluxes of energy and angular momentum transferred from the ring by the trapped magnetic fields as shown in Equations (7) and (8). The coupling of jet and accretion is taken into account by using the conservation equations of energy and angular momentum as follows,

$$
\begin{aligned}
& \frac{\mathrm{d}}{\mathrm{d} r}\left(\dot{M}_{\mathrm{D}} E^{\dagger}-g \cdot \Omega_{\mathrm{D}}\right)=4 \pi r\left(Q E^{\dagger}+S_{\mathrm{p}}\right) \\
& \frac{\mathrm{d}}{\mathrm{d} r}\left(\dot{M}_{\mathrm{D}} L^{\dagger}-g\right)=4 \pi r\left(Q L^{\dagger}+H_{\mathrm{p}}\right),
\end{aligned}
$$

where $g$ is the viscous torque in the disk, and $\Omega_{\mathrm{D}}=\Omega_{\mathrm{K}}$, and the quantities $E^{\dagger}$ and $L^{\dagger}$ are the specific energy and angular momentum given by Bardeen, Press \& Teukolsky [24].

Incorporating Equations (8)-(10), we have the radiation flux from disk as follows,

$$
\begin{aligned}
& F(r)=Q_{\mathrm{DA}} \\
& -\frac{2 f_{\mathrm{p}}}{r} \frac{-\mathrm{d} \Omega_{\mathrm{D}} / \mathrm{d} r}{\left(E^{\dagger}-\Omega_{\mathrm{D}} L^{\dagger}\right)^{2}} \int_{r_{\mathrm{in}}}^{r}\left(E^{\dagger}-\Omega_{\mathrm{D}} L^{\dagger}\right) P_{\mathrm{gas}} r^{2} \mathrm{~d} r, \\
& g(r)=\frac{E^{\dagger}-\Omega_{\mathrm{D}} L^{\dagger}}{-\mathrm{d} \Omega_{\mathrm{D}} / \mathrm{d} r} 4 \pi r F(r),
\end{aligned}
$$

where $Q_{\mathrm{DA}}$ is the viscously dissipated energy due to disk accretion, and it reads

$$
Q_{\mathrm{DA}}=\frac{1}{4 \pi r} \frac{-\mathrm{d} \Omega_{\mathrm{D}} / \mathrm{d} r}{\left(E^{\dagger}-\Omega_{\mathrm{D}} L^{\dagger}\right)^{2}} \int_{r_{\mathrm{in}}}^{r}\left(E^{\dagger}-\Omega_{\mathrm{D}} L^{\dagger}\right) \dot{M}_{\mathrm{D}} \mathrm{d} L^{\dagger} .
$$

Inspecting Equation (11), we find that the total radiation flux $F(r)$ is less than $Q_{\mathrm{DA}}$ due to the negative contribution of the second term at RHS of this equation, and this term represents the jet effect of reducing the total radiation. So we expect that disk-corona model with trapped magnetic fields anchored at the patches can be applicable to the LH state with a quasi-steady jet observed in several BHXBs, and the detailed fits will be given in the next section.

According to typical disk-corona scenario, part of the viscously dissipated energy $Q$ is released as $Q_{\mathrm{d}}^{+}$in the disk, emitting eventually as black-body radiation and 
supplying seed photons for Comptonization of corona. The rest dissipated energy, $Q_{\text {cor }}^{+}$, heats corona and maintains its relativistic temperature via magnetic buoyancy and reconnection [25]. The quantity $Q_{\text {cor }}^{+}$is proportional to magnetic energy density and local Aflven speed, and we have

$$
Q=Q_{\mathrm{d}}^{+}+Q_{\text {cor }}^{+},
$$

where $Q_{\mathrm{d}}^{+}$and $Q_{\text {cor }}^{+}$have the same meanings as given in [17].

\section{Fitting LH State of BHXBs}

In this section we fit the LH state and its associated jet in two steps, where the BHXBs, 4U 1543-475, GX 339-4, XTE J1550-564, GRO J1655-40 and GRS 1915+105 are included. Firstly, we fit the spectral profiles of the LH state of these sources, and then we fit the relation be- tween jet power and X-ray luminosity based on the first step.

\subsection{Fitting Spectra}

At the first step the spectra of the LH state are fitted based on disk-corona model, and the code given in [17] is modified in two aspects: 1) The MC process with the closed magnetic field in [17] is replaced by the jet launching process with the patched open magnetic field as shown in Figure 1, and 2) The outer boundary of corona taken as that of the closed magnetic field in [17] is replaced by the radius $r_{\text {out }}$ as an adjustable parameter in fitting the LH state in this paper.

The main characteristics of the five BHXBs are given in Table 1, and the values of the input parameters and those of $L_{\mathrm{J}}$ and $L_{\mathrm{X}}$ are given in Table 2, where $L_{\mathrm{J}}$ and $L_{\mathrm{X}}$ denote the jet power derived from Equation (6)

Table 1. The main parameters involved in fitting the LH state of five BHXBs.

\begin{tabular}{|c|c|c|c|c|}
\hline Source & $\boldsymbol{M} / \mathbf{M}_{\odot}$ & D/kpc & $i\left(^{\circ}\right)$ & $\mathrm{a} *$ \\
\hline $4 U 1543-475$ & $9.4 \pm 1.0^{\mathrm{a}}$ & $7.5^{\mathrm{a}}$ & $20.7^{\mathrm{a}}$ & $0.7 \sim 0.85^{\mathrm{b}}$ \\
\hline GX 339-4 & $5.8 \pm 0.5^{\mathrm{c}}$ & $6.0^{\mathrm{d}}$ & $20.0^{\mathrm{e}}$ & $0.7^{\mathrm{f}}$ \\
\hline GRO J1655-40 & $6.30 \pm 0.27^{g}$ & $3.2^{\mathrm{h}}$ & $70.0^{\mathrm{i}}$ & $0.65 \sim 0.8^{\mathrm{b}}$ \\
\hline XTE J1550-564 & $9.7 \sim 11.6^{\mathrm{j}}$ & $5.6^{\mathrm{j}}$ & $73.1^{\mathrm{j}}$ & $0.76 \pm 0.01^{\mathrm{b}}$ \\
\hline GRS $1915+105$ & $10 \sim 18^{\mathrm{k}}$ & $11 \sim 12^{\mathrm{k}}$ & $70 \pm 2^{1}$ & $0.98 \sim 1^{\mathrm{b}}$ \\
\hline
\end{tabular}

${ }^{\mathrm{a}}[26] ;{ }^{\mathrm{b}}[27] ;{ }^{\mathrm{c}}[28] ;{ }^{\mathrm{d}}[29] ;{ }^{\mathrm{e}}[30] ;{ }^{\mathrm{f}}[31] ;{ }^{\mathrm{g}}[32] ;{ }^{\mathrm{h}}[33] ;{ }^{\mathrm{i}}[34] ;{ }^{\mathrm{j}}[35] ;{ }^{\mathrm{k}}[2] ;{ }^{\mathrm{l}}[36]$.

Table 2. Fitting results of X-ray luminosity and jet power for five BHXBs.

\begin{tabular}{|c|c|c|c|c|c|c|}
\hline \multirow[t]{2}{*}{ Sources } & \multicolumn{4}{|c|}{ Input parameters } & \multicolumn{2}{|c|}{ Fitting results } \\
\hline & M & $\mathrm{a}_{*}$ & $\dot{m}$ & $r_{\text {out }}$ & $L_{\mathrm{J}}$ & $L_{\mathrm{x}}$ \\
\hline \multirow{4}{*}{ 4U 1543-475 } & 10.4 & 0.7 & 0.005 & 55.8 & 0.00067 & 0.0046 \\
\hline & 10.4 & 0.85 & 0.004 & 46.1 & 0.00076 & 0.0047 \\
\hline & 8.4 & 0.7 & 0.006 & 55.8 & 0.00077 & 0.0054 \\
\hline & 8.4 & 0.85 & 0.005 & 46.1 & 0.0009 & 0.0056 \\
\hline \multirow{2}{*}{ GX 339-4 } & 6.3 & 0.7 & 0.02 & 62.34 & 0.0019 & 0.022 \\
\hline & 5.3 & 0.7 & 0.025 & 62.34 & 0.0022 & 0.027 \\
\hline \multirow{4}{*}{ GRO J1655-40 } & 6.57 & 0.8 & 0.05 & 30.5 & 0.0048 & 0.013 \\
\hline & 6.57 & 0.65 & 0.06 & 37.5 & 0.00432 & 0.0127 \\
\hline & 6.03 & 0.8 & 0.05 & 30.5 & 0.0048 & 0.013 \\
\hline & 6.03 & 0.65 & 0.06 & 40.4 & 0.00429 & 0.014 \\
\hline \multirow{4}{*}{ XTE J1550-564 } & 11.6 & 0.77 & 0.017 & 55.9 & 0.0019 & 0.009 \\
\hline & 11.6 & 0.75 & 0.016 & 66.1 & 0.0018 & 0.01 \\
\hline & 9.7 & 0.77 & 0.019 & 66.4 & 0.0021 & 0.012 \\
\hline & 9.7 & 0.75 & 0.020 & 66.1 & 0.0021 & 0.012 \\
\hline \multirow{4}{*}{ GRS $1915+105$} & 10 & 0.98 & 0.35 & 69.78 & 0.15 & 0.3 \\
\hline & 10 & 0.998 & 0.3 & 51.95 & 0.216 & 0.335 \\
\hline & 18 & 0.98 & 0.25 & 50.0 & 0.098 & 0.21 \\
\hline & 18 & 0.998 & 0.18 & 35.86 & 0.11 & 0.215 \\
\hline
\end{tabular}


and the X-ray luminosity, respectively. The spectral profiles of the LH state are shown in Figure 2. It is noted that the luminosities and accretion rates are given in terms of Eddington luminosity, $1.25 \times 10^{38}\left(\mathrm{M} / \mathrm{M}_{\odot}\right) \mathrm{erg} / \mathrm{s}$, and the disk radius is given in unit of $R_{\mathrm{g}}=G M / c^{2}$.

It is noted that the spectral profiles of the LH states of thefive BHXBs given in Figure 2 are in good agreement with the observation data given in Figure 4.11 of [2].

\subsection{Relation between $X$-Ray Luminosity and Jet Power}

At the second step in fitting the LH state we check the relation between the jet power and the X-ray luminosity as follows,

$$
L_{\mathrm{J}}=A_{\text {steady }} L_{\mathrm{X}}^{0.5} \text {. }
$$

This relation was first proposed by Fender, Gallo \& Jonker [37], and the coefficient $A_{\text {steady }}$ varies between
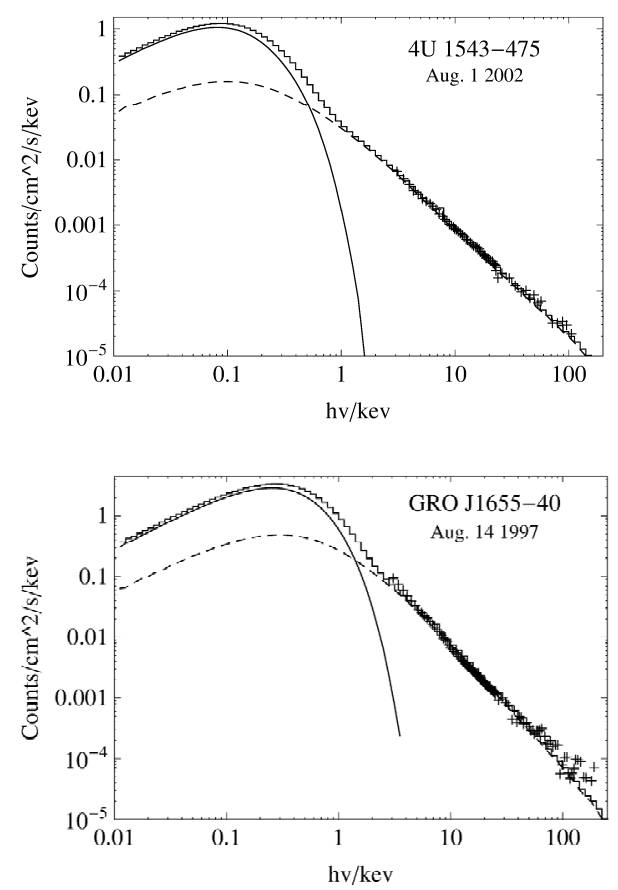

$6 \times 10^{-3}$ and $0.3[38,39]$. In our fits $L_{\mathrm{J}}$ is regarded as the jet power $\mathrm{P}_{\text {jet }}$ given by Equation (6), and $L_{\mathrm{X}}$ can be calculated based on the spectral profiles of the LH state as shown in Figure 2.

In addition, we obtain the values of $L_{\mathrm{X}}$ for each BHXB based on the spectral profile of the LH state obtained in the first step, and the coefficient $A_{\text {steady }}$ can be determined by Equation (15), which is a constant in the fitting for each source. It turns out that the relation between $L_{\mathrm{X}}$ and $L_{\mathrm{J}}$ can be satisfied by adjusting accretion rate $\dot{m}$ and the outer boundary radius $r_{\text {out }}$ of corona. The fitting results are given in Table 3 and Figure 3.

Inspecting Table 3 and Figure 3, we find that $L_{\mathrm{J}}$ and $L_{\mathrm{X}}$ do obey Equation (15) with the coefficient $A_{\text {steady }}$ ranging between $6 \times 10^{-3}$ and 0.3 , and both quantities increase monotonically with the increasing accretion rate $\dot{m}$ as well as the increasing outer boundary $r_{\text {out }}$ of the
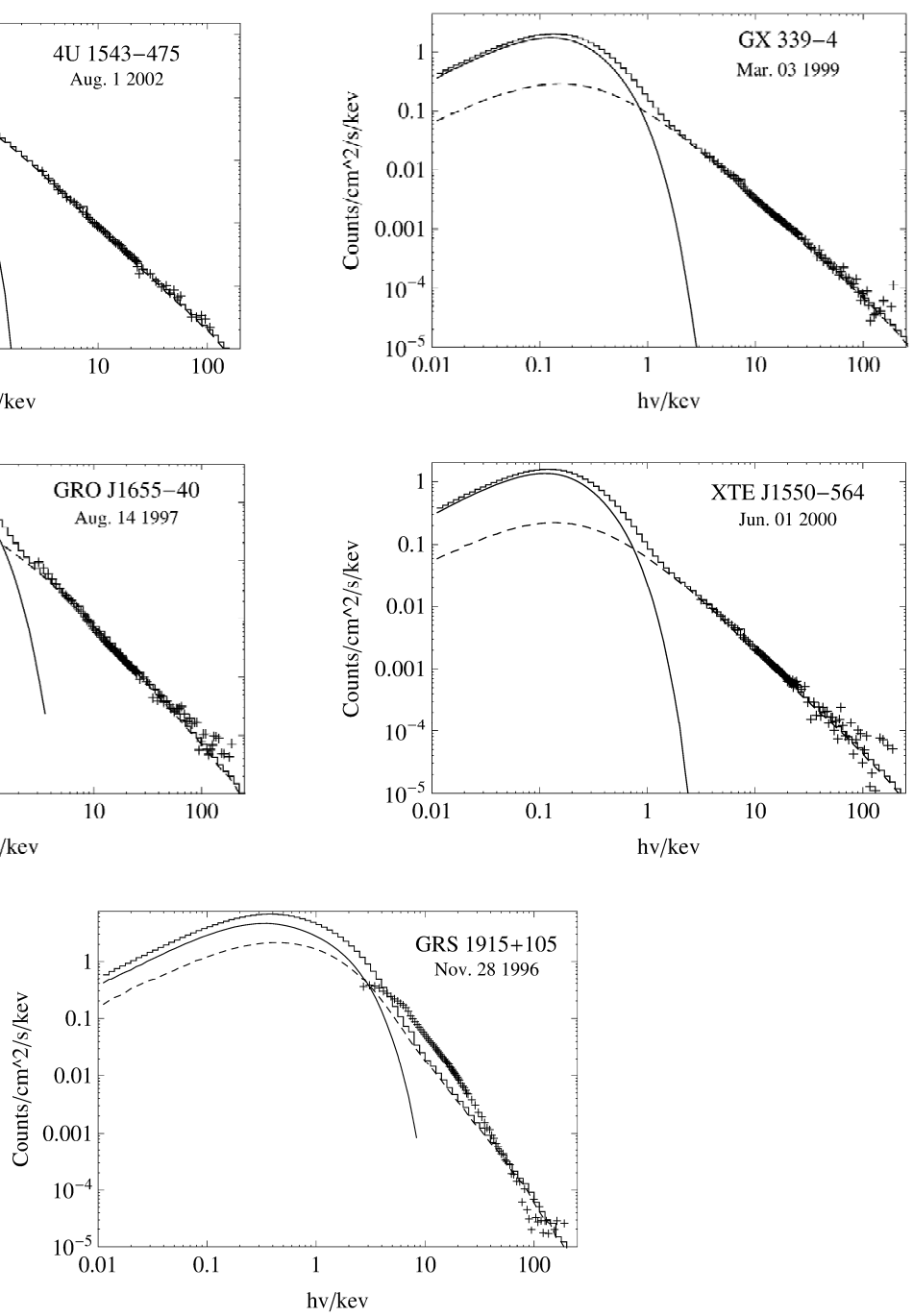

Figure 2. The spectral profiles of the LH state of five BHXBs are plotted in zigzag lines, which are superposition of thermal and power law components in solid and dashed lines, respectively. 

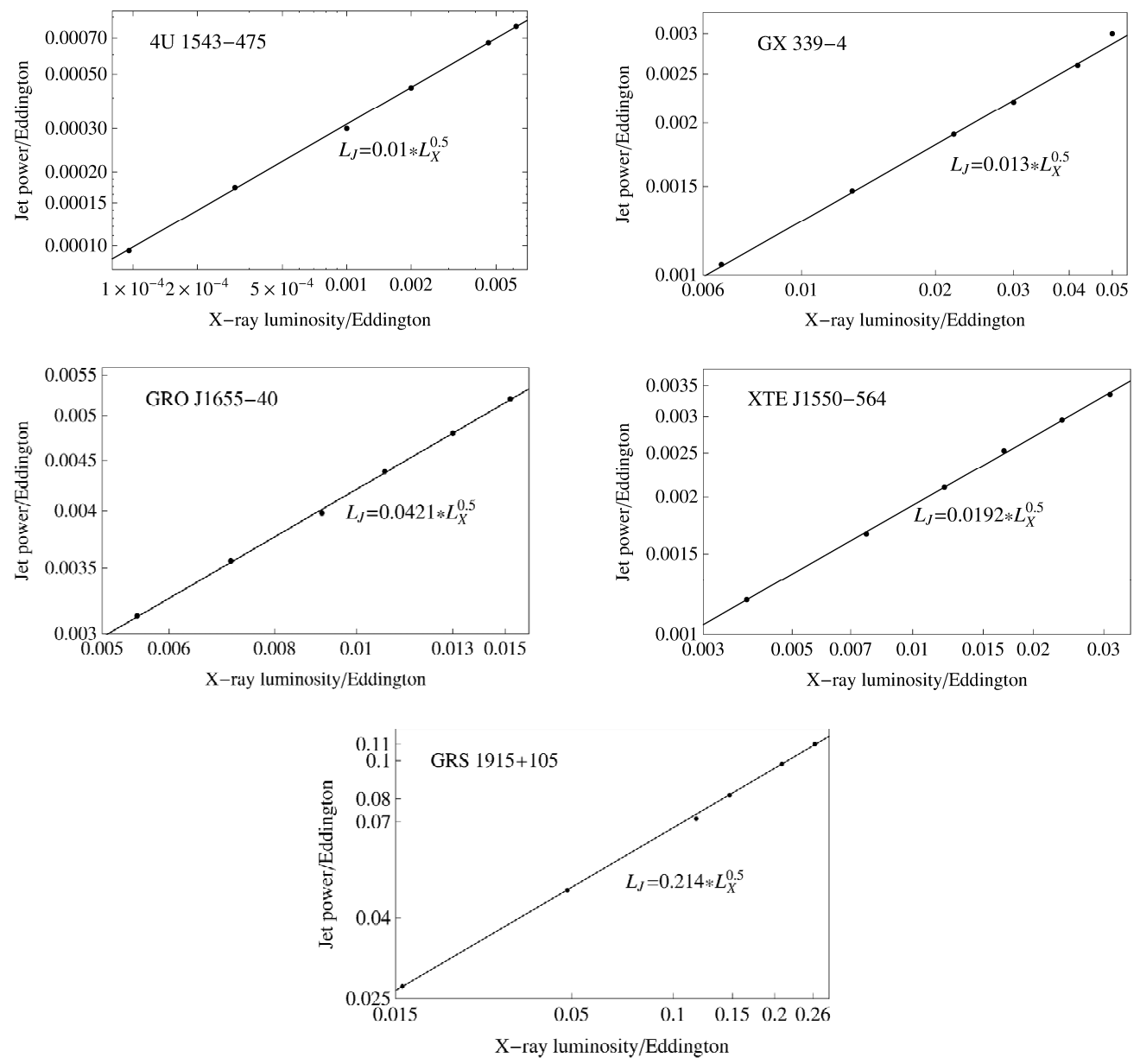

Figure 3. The relation between $L_{X}$ and $L_{J}$ in the rising phase of the LH state of five BHXBs.

Table 3. Relation between $L_{X}$ and $L_{J}$ for five BHXBs.

\begin{tabular}{|c|c|c|c|c|c|c|c|c|c|}
\hline \multirow[t]{2}{*}{ Sources } & \multicolumn{7}{|c|}{ Fitting results } & \multirow{2}{*}{$A_{\text {steady }}$} & \multirow[t]{2}{*}{$f_{p}^{\max }$} \\
\hline & $\dot{m}$ & 0.0005 & 0.001 & 0.002 & 0.003 & 0.005 & 0.006 & & \\
\hline \multirow{4}{*}{$4 U 1543-475$} & $r_{\text {out }}$ & 15.01 & 20.03 & 29.16 & 37.67 & 55.8 & 66.23 & \multirow{3}{*}{0.01} & \multirow{3}{*}{0.00016} \\
\hline & $L_{\mathrm{X}}$ & 0.0000958 & 0.0003 & 0.001 & 0.002 & 0.0046 & 0.0062 & & \\
\hline & $L_{\mathrm{J}}$ & 0.0000955 & 0.000173 & 0.0003 & 0.00044 & 0.00067 & 0.00078 & & \\
\hline & $\dot{m}$ & 0.01 & 0.015 & 0.02 & 0.025 & 0.03 & 0.035 & \multirow{4}{*}{0.013} & \multirow{4}{*}{0.00014} \\
\hline \multirow{3}{*}{ GX 339-4 } & $r_{\text {out }}$ & 35.11 & 45.99 & 62.34 & 70.62 & 95.67 & 104.8 & & \\
\hline & $L_{\mathrm{X}}$ & 0.0066 & 0.0130 & 0.022 & 0.03 & 0.0418 & 0.05 & & \\
\hline & $L_{\mathrm{J}}$ & 0.00105 & 0.00147 & 0.0019 & 0.0022 & 0.0026 & 0.003 & & \\
\hline \multirow{4}{*}{ GRO J1655-40 } & $\dot{m}$ & 0.03 & 0.035 & 0.04 & 0.045 & 0.05 & 0.055 & \multirow{4}{*}{0.0421} & \multirow{4}{*}{0.0002} \\
\hline & $r_{\text {out }}$ & 22.01 & 24.08 & 26.58 & 28.03 & 30.5 & 32.44 & & \\
\hline & $L_{\mathrm{X}}$ & 0.0055 & 0.0071 & 0.0091 & 0.0108 & 0.013 & 0.0152 & & \\
\hline & $L_{\mathrm{J}}$ & 0.00313 & 0.00356 & 0.00398 & 0.00439 & 0.0048 & 0.0052 & & \\
\hline \multirow{4}{*}{ XTE J1550-564 } & $\dot{m}$ & 0.007 & 0.012 & 0.017 & 0.022 & 0.027 & 0.032 & \multirow{4}{*}{0.020} & \multirow{4}{*}{0.00012} \\
\hline & $r_{\text {out }}$ & 32.93 & 46.09 & 55.9 & 70.85 & 88.29 & 105.3 & & \\
\hline & $L_{\mathrm{X}}$ & 0.00215 & 0.0051 & 0.009 & 0.0144 & 0.02 & 0.0265 & & \\
\hline & $L_{\mathrm{J}}$ & 0.00093 & 0.00146 & 0.0019 & 0.0024 & 0.00285 & 0.00328 & & \\
\hline \multirow{4}{*}{ GRS $1915+105$} & $\dot{m}$ & 0.1 & 0.15 & 0.2 & 0.22 & 0.25 & 0.27 & \multirow{4}{*}{0.214} & \multirow{4}{*}{0.00019} \\
\hline & $r_{\text {out }}$ & 3.884 & 5.382 & 11.55 & 16.97 & 50.0 & 225.4 & & \\
\hline & $L_{\mathrm{X}}$ & 0.0157 & 0.0485 & 0.117 & 0.0818 & 0.098 & 0.11 & & \\
\hline & $L_{\mathrm{J}}$ & 0.0268 & 0.047 & 0.0713 & 0.147 & 0.21 & 0.263 & & \\
\hline
\end{tabular}


corona. These results are consistent with the rising phase of transient outburst of the BHXBs.

This relation can be understood roughly in the context of our model. The X-ray luminosity $L_{\mathrm{X}}$ arises from the gravitational energy of the accreting matter released in the disk accretion, and the jet power $L_{\mathrm{J}}$ comes from the kinetic energy of the outflowing particles trapped in the open magnetic field lines. In fact, the two kinds of luminosities have the same origin: The gravitational energy of the matter in accretion disk. The positive exponent in the correlation implies that the both luminosities increase with the accretion rate during the LH state, because the both come from accretion process. However, the reason for the index $\sim 0.5$ remains unclear, since it involves complicated energy conversion among accretion disk, corona and outflow.

\section{A Scenario of State Transition in BHXBs}

As is well known, state transitions in BHXBs display a variety of variations not only in luminosities but also in some spectral characteristics such as hardness and spectral index. The complexity is particularly attractive in the transition from hard to soft states, with which different remarkable phenomena are associated. According to [38] and [1] the transition from hard to soft states can be depicted in a more detailed way, e.g. the transition from $\mathrm{LH}$ to HIM and to SIM states occurs successively, being associated with quasi-steady jet, episodic jet and QPOs, respectively. A jet line in HID indicates the dividing line between HIM and SIM states, and the most confusing behavior in this transition is the occurrence of episodic, relativistic jets across the jet line.

The fundamental feature in one outburst of BHXBs is the variation of the luminosity. As shown in HID these sources always start from "quiescence" of very low luminosity, and then they transit from LH to HIM states with the increasing luminosity. Afterwards the BHXBs evolve from HIM to SIM, and from SIM to HS states with luminosity remaining roughly unchanged. Then these sources transit from HS to LH states and finally return to "quiescence" with the decreasing luminosity. The variation of the X-ray luminosity is interpreted naturally by the corresponding variation of accretion rate, which is widely regarded as the first parameter for governing the state transition of BHXBs. However, accretion rate is not only parameter, since some evolution features, such as a hysteretic behavior in the state transition of BHXBs, namely from hard to soft, takes place at higher luminosities than the reverse transition later in the outburst, cannot be interpreted by the variation of accretion rate $[38,40,41]$.

It was suggested by [20] that the size of the central magnetic flux bundle can be identified with the second parameter for determining X-ray spectral states of
BHXBs and the presence of relativistic outflows. Very recently, King et al. [42] pointed out that the magnetic field might be primarily toroidal in the soft state, but primarily poloidal in the hard state. In fact, both the accumulation of the patched magnetic flux in the inner disk and the change between toroidal and poloidal magnetic fields can be regarded as evolution of magnetic field configuration. Thus we suggest that magnetic field configuration on the accretion disk is the second parameter for governing the state transition of BHXBs. This viewpoint can be interpreted roughly as follows.

By analogy with the coronal mass ejection Yuan et al. [43] proposed a MHD model for interpreting the episodic ejections from BHXBs, and GRO J1655-40, XTE J1550564 and GRS $1915+105$ are involved. It turns out that the magnetic field configuration for launching the episodic jet contains both open and closed field lines in the inner accretion flow as shown in Figure 1 of [43]. In addition, we notice that the three BHXBs with episodic ejections are exactly those with 3:2 high-frequency QPO pairs observed in SIM state (or SPL state), which are fitted by invoking closed magnetic field configuration with disk corona model in [18]. We also notice that the transitions from LH to HIM states and then to SIM state are associated with the transitions from open magnetic field configuration for LH state (this paper) to open with closed one for HIM state [43] and to closed one for SIM state [18]. Coincidentally, the three BHXBs, GRO J1655-40, XTE J1550-564 and GRS $1915+105$ are all involved in the three states, for which the fits are all given in the frame of convective turbulence of MHD. The correlation of magnetic field configurations with the transition from hard to soft states is illustrated in Figure 4.

Although the origin of the magnetic field in BHXBs remains unclear, it is most probably related to accretion process in the following aspects.

1) Seed magnetic field is regarded as one of the possibilities of the origin of the magnetic field, and seed magnetic field is brought from a companion in accretion process;

2) Seed magnetic field can be amplified via dynamo mechanism, and this mechanism arises from differential rotation of accretion disk;

3) Large-scale magnetic field might be generated by toroidal electric current, and this kind of current exist probably in the accretion due to total deviation from electric neutrality in accreting plasma coming from its companion.

Combining the above scenario of transition from hard to soft states with the prevailing scenario of transition from "high soft" state return to LH state, we obtain a complete q-shaped pattern in counter-clockwise direction consisting of upper and lower branches in HID as follows.

1) The upper branch of the q-shaped pattern (from 

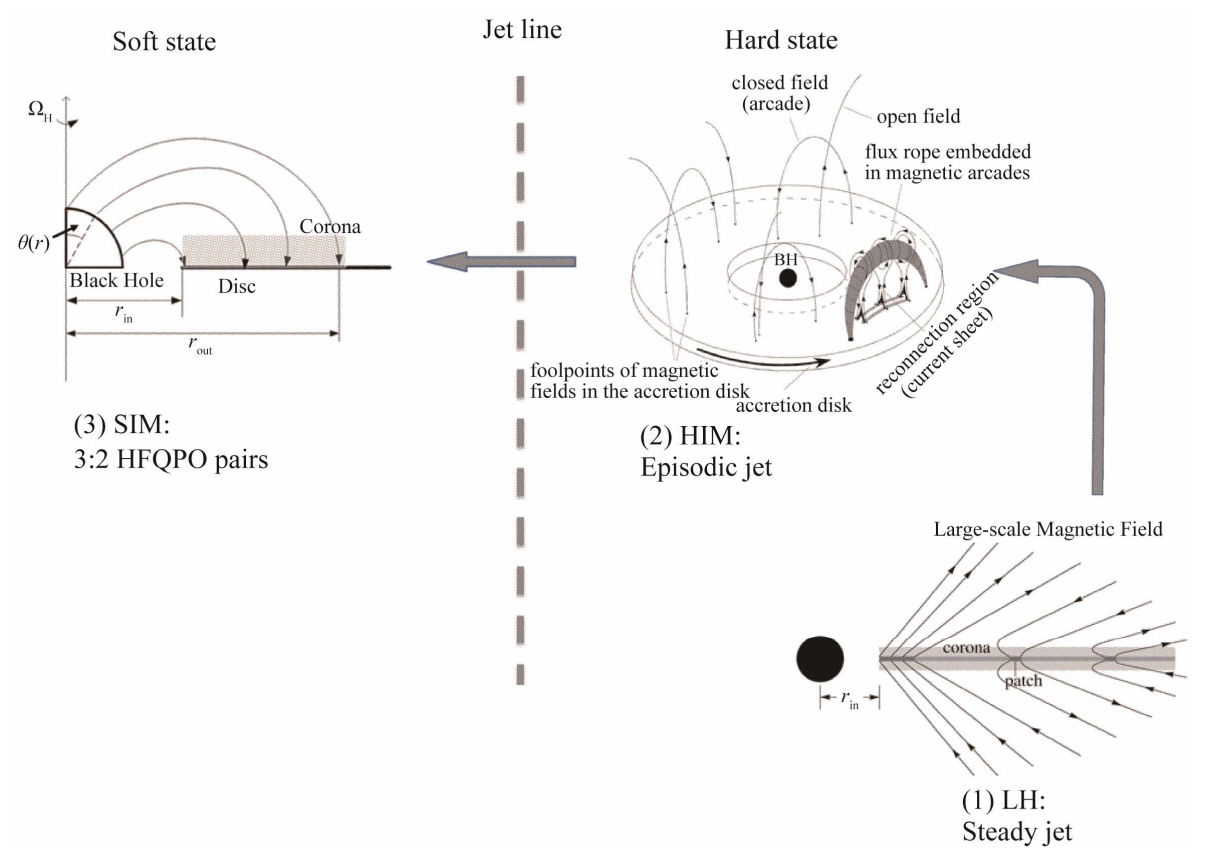

Figure 4. Illustration of correlation of magnetic field configurations with the transition from hard to soft states in BHXBs, where the magnetic field configurations corresponding to HIM and SIM states are adopted from [43] and [18], respectively.

right to left): "quiescence" $\rightarrow$ LH state $\rightarrow$ HIM state $\rightarrow$ SIM state;

2) The lower branch of the q-shaped pattern (from left to right): Soft state $\rightarrow$ LH state $\rightarrow$ "quiescence".

The scenario corresponding to the lower branch is described in [2] as follows.

Soft state (thin disk extends close to ISCO)

$\rightarrow$ LH state (a truncated thin disk with an inner ADAF)

$\rightarrow$ "quiescence" (accretion rate decreases to very low level).

However, the difference of X-ray luminosities between the upper and lower branches remains an open question.

\section{Discussion}

In this paper, we fit the spectral profiles of five BHXBs in the LH state associated with the quasi-steady jets by introducing the large-scale patched magnetic fields into disk-corona model. It turns out that a quasi-steady jet does associate with the LH state, and the relation between the jet power and the X-ray luminosity does hold based on our model.

It is noted that the coupling of patched magnetic field with accretion disk plays an essential role in the fits. On the one hand, the patched magnetic field reduces the luminosity effectively by extracting energy from the accretion disk to drive jets, and thus it affects disk dynamics. On the other hand, this effect gives rise to a feedback to the patched magnetic field itself, reducing its outward diffusion and increasing its drift inward. The main char- acteristics of the spectral profiles of the LH state can be retained with a quasi-steady jet driven by the patched magnetic field distributed dispersedly on the disk-corona system. Some issues related to our model are addressed as follows.

\section{(1) The mechanisms of driving jet}

In this paper, the magneto-centrifugal mechanism (e.g., [13]) is adopted to drive jet via the patched field instead of the BZ process [44]. Because of the following reasons, 1) The coupling of the patched magnetic fields with the disk-corona system is realized via the magneto-centrifugal mechanism, and it is helpful to interpret the $\mathrm{LH}$ state with a quasi-steady jet; 2) As shown in Figure 1, the patched fields assumed in different direction dissipate probably in magnetic reconnection as they drift close to the innermost region of the disk, and thus the BZ process cannot work due to very few magnetic fields brought to the black hole. In addition, this configuration could provide a possible interpretation for producing episodic jets in the transition from hard state to soft state $[38,43]$.

(2) Relation between jet power and $X$-ray luminosity

From the fitting results given in Table 3 and Figure 3 we find that the relation between jet power and X-ray luminosity is fitted numerically in our model. Both jet power and X-ray luminosity increases monotonically with the increasing accretion rate and the outer boundary of the corona, and these fits are consistent with the rising phase of transient outburst of the BHXBs as shown in the X-ray hardness-intensity diagram (HID) given by [38]. These results can be roughly understood as follows. 
On the one hand, both jet power and X-ray luminosity are powered essentially by gravitational energy released in accretion process, so these two quantities increase with the increasing accretion rate. On the other hand, required by the unchanged hardness of the LH state, the outer boundary of the corona should increase to contribute more power law component via Comptonization in the corona with the increasing accretion rate.

\section{(3) The uncertainty of the fitting parameters}

The most difficult problem related to our model is how to describe the distribution and the drifting motion of the magnetic patches. The purpose of this paper is to fit the correlation of $L_{\mathrm{J}}$ with $L_{\mathrm{X}}$ rather than to fit the jet power, as we have no direct observation data of the latter. Thus we can lower the requirement for describing the 3-D jet, and incorporate the four parameters into one parameter, $f_{\mathrm{p}}=k \gamma \lambda_{r} \lambda_{\varphi}$ being taken around 0.0001 in the calculations. Fortunately, the fitting results are insensitive to the value, i.e., we can obtain the same spectral profiles with a quasi-steady jet power for $f_{\mathrm{p}}$ varying around 0.0001 . The maximum values of $f_{\mathrm{p}}$ are constrained by the requirement that the radiation flux given by Equation (11) should be non-negative, and those maximum values are shown in the rightmost column of Table 3.

The fitting results presented in this paper show that the spectral profiles in the LH state could be well fitted by disk-corona model with the inner edge of disk remains close to ISCO, thus a cool accretion disk component and a relativistically-broadened $\mathrm{Fe} \mathrm{K}$ emission line can be naturally explained. Nevertheless, we have to make some assumptions with several parameters for describing the patched magnetic fields due to lack of knowledge on "flux expulsion" effect of convective turbulence, and we hope to improve our model based on further study on convective turbulence in accretion disk.

\section{Acknowledgements}

This work is supported by the NSFC (grants 11173011, 11143001, 11103003 and 11045004), the National Basic Research Program of China (2009CB824800) and the Fundamental Research Funds for the Central Universities (HUST: 2011TS159).

\section{REFERENCES}

[1] T. M. Belloni, "Black-Hole Transients: From QPOs to Relativistic Jets," Advances in Space Research, Vol. 38, No. 12, 2006, pp. 2801-2804. doi:10.1016/j.asr.2005.10.048

[2] J. E. McClintock and R. A. Remillard, "Black Hole Binaries," In: W. Lewin and M. van der Klis., Eds., Compact Stellar X-Ray Sources, Cambridge University Press, Cambridge, 2006, pp. 157-213.
[3] A. A. Esin, J. E. McClintock and R. Narayan, "Advection-Dominated Accretion and the Spectral States of Black Hole X-Ray Binaries: Application to Nova MUSCAE 1991," The Astrophysical Journal, Vol. 489, No. 2, 1997, pp. 865-889. doi:10.1086/304829

[4] A. Esin, R. Narayan, W. Cui, J. E. Grove and S. N. Zhang, "Spectral Transitions in Cygnus X-1 and Other Black Hole X-Ray Binaries," The Astrophysical Journal, Vol. 505, No. 2, 1998, pp. 854-868. doi:10.1086/306186

[5] C. Done, M. Gierlinski and A. Kubota, "Modelling the Behaviour of Accretion Flows in X-Ray Binaries. Everything You Always Wanted to Know about Accretion but Were Afraid to Ask," The Astronomy and Astrophysics Review, Vol. 15, No. 1, 2007, pp. 1-66. doi:10.1007/s00159-007-0006-1

[6] J. M. Miller, J. Homan and G. Miniutti, "A Prominent Accretion Disk in the Low-Hard State of the Black Hole Candidate SWIFT J1753.5-0127," The Astrophysical Journal Letters, Vol. 652, 2006, pp. L113-L116. doi:10.1086/510015

[7] J. M. Miller, et al., "A Long, Hard Look at the Low/Hard State in Accreting Black Holes," The Astrophysical Journal, Vol. 653, No. 1, 2006, pp. 525-535. doi: $10.1086 / 508644$

[8] R. C. Reis, J. M. Miller and A. C. Fabian, "Thermal Emission from the Stellar-Mass Black Hole Binary XTE J1118+480 in the Low/Hard State," Monthly Notices of the Royal Astronomical Society: Letters, Vol. 395, No. 1, 2009, pp. L52-L56. doi:10.1111/j.1745-3933.2009.00640.x

[9] R. C. Reis, A. C. Fabian and J. M. Miller, "Black Hole Accretion Discs in the Canonical Low-Hard State," Monthly Notices of the Royal Astronomical Society, Vol. 402, No. 2, 2010, pp. 836-854. doi:10.1111/j.1365-2966.2009.15976.x

[10] J. Contopoulos, "A Simple Type of Magnetically Driven Jets: An Astrophysical Plasma Gun," The Astrophysical Journal, Vol. 450, 1995, pp. 616-627. doi: $10.1086 / 176170$

[11] I. Contopoulos and D. Kazanas, "A Cosmic Battery," The Astrophysical Journal, Vol. 508, No. 2, 1998, pp. 859863. doi: $10.1086 / 306426$

[12] D. Lynden-Bell, "Magnetic Collimation by Accretion discs of Quasars and Stars," Monthly Notices of the Royal Astronomical Society, Vol. 279, No. 2, 1996, pp. 389401.

[13] G. S. Bisnovatyi-Kogan and A. A. Ruzmaikin, "The Accretion of Matter by a Collapsing Star in the Presence of a Magnetic Field. II-Selfconsistent Stationary Picture," Astrophysics and Space Science, Vol. 42, No. 2, 1976, pp. 401-424. doi:10.1007/BF01225967

[14] R. D. Blandford and D. G. Payne, "Hydromagnetic Flows from Accretion Discs and the Production of Radio Jets," Monthly Notices of the Royal Astronomical Society, Vol. 199, No. 3, 1982, pp. 883-903.

[15] M. Livio, "Astrophysical Spouts: The Jet Set," Nature, Vol. 417, No. 6885, 2002, p. 125.

[16] H. C. Spruit, "Theory of Magnetically Powered Jets," In: 
H. C. Spruit, Ed., The Jet Paradigm, Lecture Notes in Physics, Springer-Verlag, Berlin, 2010, p. 233. doi:10.1007/978-3-540-76937-8_9

[17] Z. M. Gan, D. X. Wang and W. H. Lei, "A Model of Magnetically Induced Disc-Corona for Black Hole Binaries," Monthly Notices of the Royal Astronomical Society, Vol. 394, No. 4, 2009, pp. 2310-2320. doi:10.1111/j.1365-2966.2009.14518.x

[18] C. Y. Huang, Z. M. Gan, J. Z. Wang and D. X. Wang, "A Resonance Model with Magnetic Connection for 3:2 HFQPO Pairs in Black Hole Binaries," Monthly Notices of the Royal Astronomical Society, Vol. 403, No. 4, 2010, pp. 1978-1982. doi:10.1111/j.1365-2966.2009.16237.x

[19] A. Merloni and A. C. Fabian, "Coronal Outflow Dominated Accretion Discs: A New Possibility for Low-Luminosity Black Holes?" Monthly Notices of the Royal Astronomical Society, Vol. 332, No. 1, 2002, pp. 165-175. doi:10.1046/j.1365-8711.2002.05288.x

[20] H. C. Spruit and D. A. Uzdensky, "Magnetic Flux Captured by an Accretion Disk," The Astrophysical Journal, Vol. 629, No. 2, 2005, pp. 960-968.

doi:10.1086/431454

[21] Y. B. Zeldovich, "The Magnetic Field in the Two-Dimensional Motion of a Conducting Turbulent Liquid," JETP, Vol. 31, 1956, pp. 154-156.

[22] E. N. Parker, "A Kinematical Theory of Turbulent Hydromagnetic Fields," The Astrophysical Journal, Vol. 138, 1963, p. 226. doi:10.1086/147629

[23] S. A. Balbus and J. F. Hawley, "A Powerful Local Shear Instability in Weakly Magnetized Disks. I-Linear AnaLysis. II-Nonlinear Evolution," The Astrophysical Journal, Vol. 376, 1991, pp. 214-233. doi:10.1086/170270

[24] J. M. Bardeen, W. H. Press and S. A. Teukolsky, "Rotating Black Holes: Locally Nonrotating Frames, Energy Extraction, and Scalar Synchrotron Radiation," The Astrophysical Journal, Vol. 178, 1972, pp. 347-370. doi:10.1086/151796

[25] B. F. Liu, S. Mineshige and K. Shibata, "A Simple Model for a Magnetic Reconnection-Heated Corona," The Astrophysical Journal Letters, Vol. 572, 2002, pp. L173-L176. doi: $10.1086 / 341877$

[26] N. La Palombara and S. Mereghetti, "XMM-Newton Observation of 4U 1543-475: The X-Ray Spectrum of a Stellar-Mass Black-Hole at Low Luminosity," Astronomy and Astrophysics, Vol. 433, No. 3, 2005, pp. L53-L56. doi:10.1051/0004-6361:200400123

[27] R. P. Fender, E. Gallo and D. Rusell, "No Evidence for Black Hole Spin Powering of Jets in X-ray Binaries," Monthly Notices of the Royal Astronomical Society, Vol. 406, No. 3, 2010, pp. 1425-1434. doi:10.1111/j.1365-2966.2010.16754.x

[28] R. I. Hynes, D. Steeghs, J. Casares, P. A. Charles and K. O'Brien, "Dynamical Evidence for a Black Hole in GX 339-4," The Astrophysical Journal Letters, Vol. 583, 2003, pp. L95-L98. doi:10.1086/368108

[29] A. A. Zdziarski, J. Poutanen, J. Mikołajewska, M. Gierlinski, K. Ebisawa and W. N. Johnson, "A Spectral De- composition of the Variable Optical, Ultraviolet and XRay Continuum of NGC 5548," Monthly Notices of the Royal Astronomical Society, Vol. 301, No. 1, 1998, pp. 179-192. doi:10.1046/j.1365-8711.1998.02015.x

[30] J. M. Miller, et al., "Initial Measurements of Black Hole Spin in GX 339-4 from Suzaku Spectroscopy," The Astrophysical Journal Letters, Vol. 679, No. 2, 2008, pp. L113-L116. doi:10.1086/589446

[31] M. Kolehmainen and C. Done, "Limits on Spin Determination from Disc Spectral Fitting in GX 339-4," Monthly Notices of the Royal Astronomical Society, Vol. 406, No. 4, 2010, pp. 2206-2212. doi:10.1111/j.1365-2966.2010.16835.x

[32] R. Shafee, et al., "Estimating the Spin of Stellar-Mass Black Holes by Spectral Fitting of the X-Ray Continuum," The Astrophysical Journal Letters, Vol. 636, 2006, pp. L113-L116. doi:10.1086/498938

[33] S. J. Tingay, et al., "Relativistic Motion in a Nearby Bright X-Ray Source," Nature, Vol. 374, No. 6518, 1995, pp. 141-143. doi:10.1038/374141a0

[34] C. D. Bailyn, J. A. Orosz, J. E. McClintock and R. A. Remillard, "Dynamical Evidence for a Black Hole in the Eclipsing X-Ray Nova GRO J1655-40," Nature, Vol. 378, No. 6553,1995 , pp. 157-159. doi: $10.1038 / 378157 \mathrm{a} 0$

[35] J. A. Orosz, et al., "Dynamical Evidence for a Black Hole in the Microquasar XTE J1550-564," The Astrophysical Journal, Vol. 568, No. 2, 2002, pp. 845-861. doi: $10.1086 / 338984$

[36] J. Greiner, J. G. Cuby and M. J. McCaughrean, "An Unusually Massive Stellar Black Hole in the Galaxy," Nature, Vol. 414, No. 6863, 2001, pp. 522-525.

[37] R. P. Fender, E. Gallo and P. Jonker, "Jet-Dominated States: An Alternative to Advection across Black Hole Event Horizons in 'Quiescent' X-Ray Binaries," Monthly Notice of the Royal Astronomical Society, Vol. 343, No. 4, 2003, pp. L99-L103. doi:10.1046/j.1365-8711.2003.06950.x

[38] R. P. Fender, T. M. Belloni and E. Gallo, "Towards a Unified Model for Black Hole X-Ray Binary Jets," Monthly Notices of the Royal Astronomical Society, Vol. 355, No. 4, 2004, pp. 1105-1118. doi:10.1111/j.1365-2966.2004.08384.x

[39] J. Malzac, A. Merloni and A. C. Fabian, "Jet-Disc Coupling through a Common Energy Reservoir in the Black Hole XTE J1118+480," Monthly Notices of the Royal Astronomical Society, Vol. 351, No. 1, 2004, pp. 253-264. doi:10.1111/j.1365-2966.2004.07772.x

[40] S. Miyamoto, S. Kitamoto, K. Hayahida and W. Egoshi, "Large Hysteretic Behavior of Stellar Black Hole Candidate X-Ray Binaries," The Astrophysical Journal Letters, Vol. 442, No. 1, 1995, pp. L13-L16. doi: $10.1086 / 187804$

[41] T. M. Belloni, “The Jet Paradigm,” In: The Jet Paradigm: from Microquasars to Quasars, Lecture Notes in Physics, Springer-Verlag, Berlin, 2010, p. 53. doi:10.1007/978-3-540-76937-8

[42] A. L. King, et al., "An Extreme X-Ray Disk Wind in the 
Black Hole Candidate IGR J17091-3624," The Astrophysical Journal Letters, Vol. 746, 2012, p. L20. doi:10.1088/2041-8205/746/2/L20

[43] F. Yuan, J. Lin, K. W. Wu and C. H. Luis, "A Magnetohydrodynamical Model for the Formation of Episodic Jets," Monthly Notices of the Royal Astronomical Society,
Vol. 395, No. 4, 2009, pp. 2183-2188. doi:10.1111/j.1365-2966.2009.14673.x

[44] R. D. Blandford and R. L. Znajek, "Electromagnetic Extraction of Energy from Kerr Black Holes," Monthly Notices of the Royal Astronomical Society, Vol. 179, 1977, pp. 433-456. 